\title{
Peroxide dental bleaching via laser microchannels and tooth color measurements
}

\author{
Gregory Altshuler \\ Andrey Belikov \\ Alexei Skrypnik \\ Felix Feldchtein \\ Alexandra Pushkareva \\ Ksenia Shatilova \\ Igor Cernavin \\ Valery Tuchin
}




\title{
Peroxide dental bleaching via laser microchannels and tooth color measurements
}

\author{
Gregory Altshuler, ${ }^{a}$ Andrey Belikov, ${ }^{b, *}$ Alexei Skrypnik, ${ }^{b}$ Felix Feldchtein, ${ }^{c}$ Alexandra Pushkareva, ${ }^{b}$ \\ Ksenia Shatilova, ${ }^{b}$ Igor Cernavin, ${ }^{\mathrm{d}}$ and Valery Tuchin ${ }^{\mathrm{e}, \mathrm{d}, \mathrm{g}}$ \\ aIPG Medical Corporation, 377 Simarano Drive, Marlborough, Massachusetts 01752, United States \\ bSaint Petersburg National Research University of Information Technologies, Mechanics and Optics, Faculty of Laser and Light Engineering, \\ Department of Laser Technologies and Systems, 49 Kronverksky Avenue, Saint-Petersburg 197101, Russia \\ 'Medical Device Consultant, 28 Lebeaux Drive, Shrewsbury, Massachusetts 01545, United States \\ dUniversity of Melbourne, School of Dentistry, Faculty of Medicine, Dentistry and Health Sciences, 42 The Eyrie, Eaglemont, Victoria 3084, \\ Australia \\ eSaratov National Research State University, Research-Education Institute of Optics and Biophotonics, 83 Astrakhanskaya Street, \\ Saratov 410012, Russia \\ 'Institute of Precision Mechanics and Control of Russian Academy of Sciences, 24 Rabochaya Street, Saratov 410028, Russia \\ 9National Research Tomsk State University, Laboratory of Biophotonics, 36 Lenin's Avenue, Tomsk 634050, Russia
}

\begin{abstract}
The aim of this study was to use microchannels drilled by an Er:YAG laser into a human tooth through the enamel into the dentin for direct injection of hydrogen peroxide (HP) to produce a minimally invasive, rapid, tooth bleaching effect. The experiments were conducted in vitro. Five microchannels with a diameter of $\sim 200 \mu \mathrm{m}$ and a depth of $\sim 2 \mathrm{~mm}$ were drilled through the palatal side of a human tooth crown using the microbeam of an Er:YAG-laser with a wavelength of $2.94 \mu \mathrm{m}$. After injection of an aqueous solution of 31\%-HP through the microchannels, the tooth color was evaluated using a VITA shade guide and International Commission on Illumination $L * a b$ color parameters. A tooth model used for the evaluation of the distribution of HP concentration was created and the amount of HP which can be injected into tooth dentin to bleach it safely was estimated. Injection of $1.5 \pm 0.1 \mathrm{~mm}^{3}$ of $31 \%-\mathrm{HP}$ into the tooth led to noticeable bleaching within $3 \mathrm{~h}$ and significant improvement of tooth color within 24 h. () 2016 Society of Photo-Optical Instrumentation Engineers (SPIE) [DOI: 10.1117/1.JBO.21.12.125001]
\end{abstract}

Keywords: Er:YAG-laser; microchannels; tooth bleaching; color parameters; lightness.

Paper 160563R received Aug. 29, 2016; accepted for publication Nov. 10, 2016; published online Dec. 7, 2016.

\section{Introduction}

Tooth color is determined by the spectral signature of endogenous or exogenous chromophores and their location within tooth structures. Two types of discoloration, extrinsic and intrinsic, can affect tooth appearance. The extrinsic is due to staining of the tooth surface by food products, such as tea, coffee, wine, tobacco, and so on. This staining can be effectively minimized by regular and correct home tooth brushing, or removed by professional cleaning in the dental office.

Intrinsic staining occurs as a result of natural pigmentation or blood and medical or chemical material penetrating into tooth dentin. ${ }^{1-3}$ Discoloration also occurs due to the release of disintegrated by-products into the surrounding dentin from the dental pulp by bacterial, mechanical, or chemical irritants. ${ }^{1}$

Nathoo $^{2}$ has shown that intrinsic staining can be divided into pre-eruptive and posteruptive types. Endemic fluorosis, tetracycline stains, dentinogenesis imperfecta, amelogenesis imperfecta, and hematologic disorders are examples of pre-eruptive intrinsic staining. Posteruptive intrinsic staining tends to be due to factors such as pulpal hemorrhage, secondary and tertiary dentin deposition, pulp stones, metal release from amalgam restorations, and incomplete obturation of the pulp chamber.

Intrinsic staining is more difficult to treat and peroxide-based tooth bleaching [hydrogen peroxide (HP) or one of its precursors such as sodium perborate or carbamide peroxide, which are strong oxidants] is typically used. ${ }^{1,4}$

*Address all correspondence to: Andrey Belikov, E-mail: avbelikov@ gmail.com
The mechanisms of peroxide-based tooth bleaching are not well understood. They differ according to the type of discoloration involved and the chemical and physical conditions at the site of the chemical reaction. ${ }^{4}$ In general, these mechanisms include the oxidation of chromophores located within enamel and dentin and the degradation of the extracellular matrix. As a result of oxidation, the $\pi$-conjugated electronic systems of the chromophore molecules are broken, and their absorption bands are shifted from the visible to the UV region, resulting in a whiter appearance of the teeth.

Unfortunately, peroxide-based bleaching technology has a number of drawbacks, imposing serious limitations and concerns at application. ${ }^{5-10}$ Raman and fluorescence spectroscopy has shown that compounds with a concentration of HP of about $30 \%$ may cause adverse effects to the mineral and organic matter of human tooth enamel, reducing enamel microhardness. ${ }^{5}$ The drawbacks also include local effects on the oral mucosa including gingivitis, ${ }^{6,7}$ erosion, and abrasion of enamel and dentin, ${ }^{7,8}$ modification of dentinal permeability with posttreatment transient tooth sensitivity, ${ }^{7,9}$ possible effects on pulpal tissues and dental restorations, ${ }^{5,10}$ and resorption in the cervical area of the teeth. ${ }^{5}$ Repeated treatment, which is usually required to reach the desired bleaching condition, adds to these adverse effects. ${ }^{5}$

To improve the efficacy of the oxidizer, to make the technology safer and faster, different physical and chemical enhancers for HP activation and better diffusion have been suggested and studied. ${ }^{11-21}$ For example, teeth with an applied bleaching agent

1083-3668/2016/\$25.00 @ 2016 SPIE 
were subjected to local heating ${ }^{11}$ or intensive white light and light-emitting diode, or laser. ${ }^{11-18}$ Suemori et al. ${ }^{16}$ and Calatayud et al. ${ }^{18}$ have shown that effective bleaching of blood stained dentin was achieved using a chemical composition containing only $3.5 \%$-HP and titanium dioxide photocatalytic nanoparticles at $405 \mathrm{~nm}$-diode laser excitation.

A bleaching agent can also be applied externally to vital teeth-known as vital bleaching, or internally within the pulp chamber of nonvital teeth-known as nonvital bleaching. ${ }^{22}$ The penetration of HP molecules when applied externally to tooth structures has not been well controlled until now, since $^{23}$ the effect largely depends on the individual aspects of the tooth. Ubaldini et al. ${ }^{23}$ have shown that permeation is not merely a physical passage through enamel interprismatic spaces into the dentinal tubules, but that HP diffusion dynamics present a concentration gradient determined by the chemical affinity of the HP molecules to each specific dental tissue.

Altshuler et al. ${ }^{24}$ found that the chemical interaction of HP with enamel is minor, whereas the interaction with dentin is noticeable. Intrinsic bleaching can be performed when the pulp is removed and a large access cavity is cut, to place a medium with HP for a prolonged exposure. ${ }^{22}$ It is much more effective, because the HP molecules release active oxygen and other radicals in the immediate vicinity of the target chromogenic molecules. This technique cannot, however, be used for vital teeth.

The importance of the use of a high-quality Er:YAG laser beam for well controlled intrinsic bleaching of vital teeth was discussed by Altshuler et al. ${ }^{24}$ and Belikov et al. ${ }^{25}$ The aim of this paper is to prove the feasibility of tooth bleaching using an aqueous solution of HP via microchannels drilled by an Er:YAG laser through the enamel into the dentin in a manner which will not damage the pulp.

The concentration of HP that will not damage the pulp is described using the results of Bowles et al., ${ }^{26}$ who showed that a direct application of up to $2.5 \% \mathrm{HP}$ when injected into pulpal extract does not inhibit its enzymes at normal body temperature. On this basis, it can be concluded that if the concentration of HP in dentin at the pulpal surface does not exceed $1 \%$ during the entire bleaching procedure, no adverse effects should occur.

This study was conducted on freshly extracted human teeth in vitro using an aqueous solution of $31 \%$-HP. The bleaching process was allowed to continue for a period of $48 \mathrm{~h}$ and the color changes were determined using the VITA shade guide and International Commission on Illumination (CIE) $L * a b$ color parameter determination: $L$ (lightness), $a$ (redness), and $b$ (yellowness). A tooth model for numerical simulation of $31 \%$-HP aqueous solution diffusion into the tooth dentin through laser drilled microchannels within the tooth crown is presented.

\section{Materials and Methods}

\subsection{In Vitro Experiment}

The teeth used for the experiments were intact human maxillary central incisors, extracted as these teeth were to be replaced with implant supported crowns. The extracted teeth were then stored in an aqueous solution of $0.1 \%$-thymol for no $>4$ weeks. The age of the patients was 45 to 50 years (ITMO, LTBMO IRB approval \#04 at 23/04/2015). Immediately prior to treatment, the teeth were taken from the thymol solution, the crowns were cleaned using a mixture of abrasive pumice powder (medium coarse \#3, Kerr Dental) and water for $60 \mathrm{~s}$ with a brush (RA Junior Cup White Bristle Martins) in a slow-speed drill (8000 rpm).

After cleaning, 1 to $2 \mathrm{~mm}$ of the apex of tooth root was sectioned using a diamond disc to facilitate fixation of the tooth into a stainless steel container, black in color. The tooth was then cemented into this container using a dental cement (Meron, Combipack, VOCO GmbH, Germany) and both tooth and container were placed into a $0.1 \%$-thymol solution for a period of 5 to $10 \mathrm{~min}$. The container with the tooth was taken from the thymol solution and fixed into a holder for photographing. The holder was designed so that the tooth was always kept in the same position to provide comparable photographs before and after tooth processing. The appropriately matching tooth from the shade guide (VITAPAN ${ }^{\circledR}$ classical, VITA Zahnfabrik H.Rauter Gmb\&Co.KG, Germany) was placed next to the tooth under observation, into a similar holder.

A digital camera (Nikon D80) with a flash lamp (Sigma EM140DG) was placed at a distance of $50 \mathrm{~cm}$ from the holder. The camera had an external power supply, remote control, and USB cable for online reading via a computer. All camera and flash settings (aperture, focus, exposure, white balance, ISO, and so on) were set manually for each photograph. A standard $18 \%$ gray card was placed in the field of view for all photographs for digital image calibration and verification as described by Bengel. $^{27}$

The camera, flash lamp, tooth in its container, VITA shade guide tooth, and $18 \%$ gray card in its container were covered with a black velvet cloth to eliminate ambient light. They were photographed then the container with the tooth was removed from its holder, and laser drilling was carried out. Five microchannels with a diameter of $\sim 200 \mu \mathrm{m}$ and a depth of $\sim 2 \mathrm{~mm}$ (see Fig. 1) were drilled through the palatal surface of the tooth crown using an Er:YAG laser with a wavelength of $2.94 \mu \mathrm{m}$ and a beam propagation ratio of $1.5 \pm 0.1$. The beam propagation ratio indicates how close the laser beam is to being in the fundamental Gaussian mode, which allows for the smallest spot size and minimal beam divergence. ${ }^{28}$
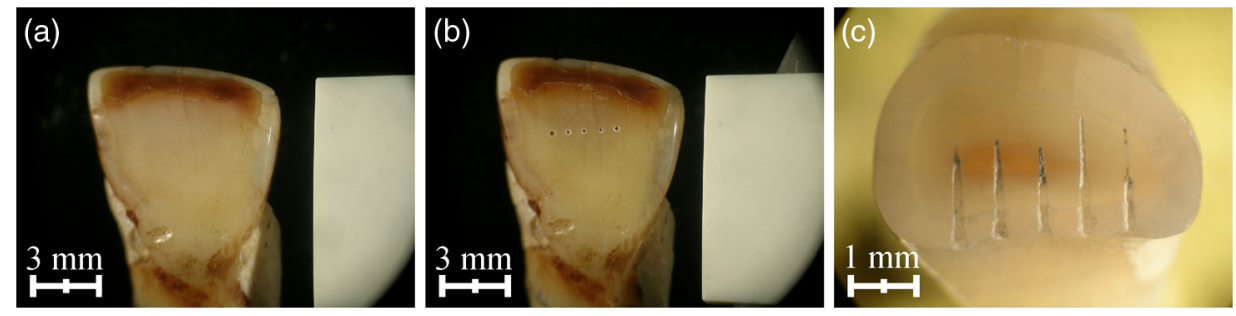

Fig. 1 Photographs of a tooth crown (a) before and (b) after laser drilling, and (c) a cross-sectional view of the drilled microchannels after tooth cut along channels. 
The laser beam was focused onto the palatal side of the crown using a lens with a focal length of $\sim 38 \mathrm{~mm}$ to drill the microchannel. The target area of the crown was irrigated with a drop of water of $\sim 1 \mathrm{~mm}^{3}$ immediately prior to laser drilling. Each microchannel was formed using $\mathrm{M}^{2}$ laser technology, ${ }^{29}$ with 80 laser pulses at a repetition rate of $1 \mathrm{~Hz}$ $(F=1 \mathrm{~Hz})$. The free-running laser pulse duration of $\tau_{p}=$ $150 \pm 15 \mu \mathrm{s}$, pulse energy of $E_{p}=28.0 \pm 0.5 \mathrm{~mJ}$, and the beam diameter at $e^{-2}$ intensity level of $120 \pm 10 \mu \mathrm{m}$ were used. The distance between the microchannel centers was $\sim 500 \mu \mathrm{m}$.

After laser drilling, the container with the tooth was placed under a microscope at 16× magnification and 96\%-ethyl alcohol was injected into the microchannels for 20 to $30 \mathrm{~s}$ using a syringe with a needle, with an external diameter of $110 \mu \mathrm{m}$ (World Precision Instruments, Inc.), to clean the microchannels from any debris. They were then dried using air and each one was filled with $31 \%$-HP heated to $40^{\circ} \mathrm{C}$. A needle with an external diameter of $110 \mu \mathrm{m}$ (World Precision Instruments, Inc.) was used to fill the microchannels with the HP. The needle was pushed to the bottom of microchannel so that the HP could displace the air trapped inside the channel and minimize the probability of air bubbles. A microscope was used to visually check that each microchannel was filled correctly, with injection ceasing once it was assessed that the microchannel was full. The total amount of HP at $31 \%$ concentration that was used to fill the five microchannels was $1.5 \pm 0.1 \mathrm{~mm}^{3}$.

The container with the tooth was then placed in a $0.1 \%$-thymol solution for $1 \mathrm{~min}$ and fixed into the appropriate holder to take the photograph of the tooth, VITA shade guide, and gray card. Photographs were taken at 3, 24, and $48 \mathrm{~h}$ after $31 \%$-HP injection. The containers with the teeth were stored in a $0.1 \%$ thymol solution at a temperature of $37^{\circ} \mathrm{C}$ between photographs.

Tooth shade was evaluated using the VITA shade guide (VITAPAN® classical, VITA Zahnfabrik H. Rauter Gmb\&Co. $\mathrm{KG}$, Germany) prior to and at 3, 24, and $48 \mathrm{~h}$ after HP-injection. All images were processed using Adobe Photoshop CS6 following the method described by Bengel ${ }^{27}$ and color CIE $L * a b$ color parameters: $L$ (lightness), $a$ (redness), and $b$ (yellowness) were calculated for the VITA shade guide and teeth. The differential color parameters $\Delta(\mathrm{CIE} L * a b)$ could then be calculated for the 3,24 , and $48 \mathrm{~h}$ time periods after HP-injection, to compare the difference in appearance between the commencement and the various time periods.

\subsection{Statistical Analysis}

Fifteen human maxillary central incisor teeth were used for the study. Five teeth which were not treated by laser and peroxide were used as the control group (C). Five teeth which were subjected to laser drilling only were used as the test1 group (Tx1). Five teeth processed with laser drilling and exposed to $31 \%$-HP were used as a test 2 group (Tx2). The software package StatGraphics Plus 2.1 (Statistical Graphics Corp.) was used for statistical data processing. The means and confidence interval for $\Delta$ of CIE $L * a b$ color parameters in the control $(\mathrm{C})$ and test (Tx1 and Tx2) groups were calculated. The KolmogorovSmirnov test to estimate the statistical difference for $\Delta$ of CIE $L * a b$ color parameters between the control (C) and test (Tx1 and Tx2) groups, with $p$-value $<0.05$ was used.

\section{Results}

The typical digital images of the VITA shade guide tooth, 18\% gray card, and treated (Tx2) tooth (sample \#1Tx2) before and after injection of 31\%-HP through the microchannels drilled by Er:YAG laser radiation at varying time intervals are shown in Fig. 2.

The results of the shade evaluation using the standard VITA shade guide are shown in Fig. 3.

The CIE $L * a b$ color parameters describing $L$ (lightness), $a$ (redness), and $b$ (yellowness) of the tooth that were measured for the samples from the control (C) and test (Tx1 and Tx2) groups and presented in Table 1.
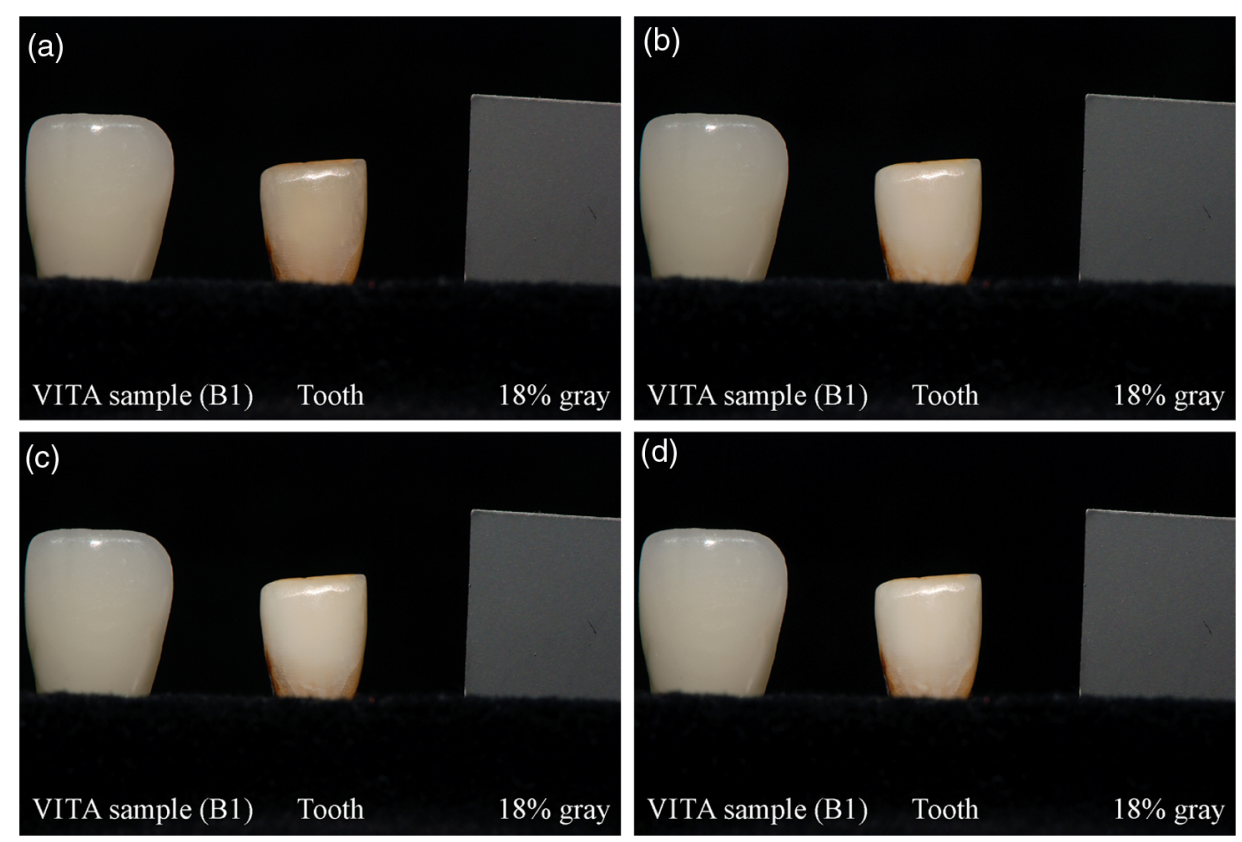

Fig. 2 Photographs of the VITA shade guide tooth, Tx tooth (sample \#1Tx), and $18 \%$ gray card: (a) before, (b) at $3 \mathrm{~h}$, (c) at $24 \mathrm{~h}$, and (d) at $48 \mathrm{~h}$ after injection of $1.5 \pm 0.1 \mathrm{~mm}^{3}$ volume of $31 \%-\mathrm{HP}$ through microchannels drilled by a $2.94-\mu \mathrm{m}$-Er:YAG laser. 


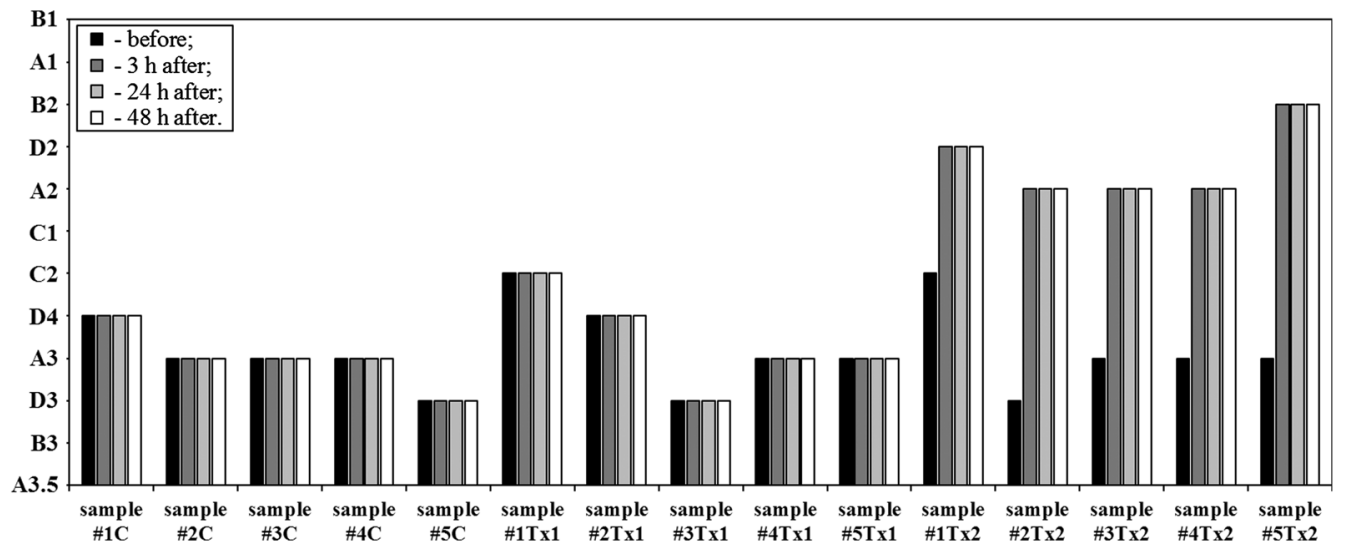

Fig. 3 Results of shade evaluation using the VITA shade guide for the control (C) and test teeth (Tx) samples before bleaching, at 3,24 , and $48 \mathrm{~h}$ after injection of $1.5 \pm 0.1 \mathrm{~mm}^{3}$ volume of $31 \%-\mathrm{HP}$ through the microchannels drilled by Er:YAG laser radiation.

The differential color parameters $\Delta(\mathrm{CIE} L * a b)$ for the samples from the control group (C) and test (Tx1 and Tx2) groups quantifying changes in the CIE $L * a b$ color parameters of the tooth before treatment and for the varying times after treatment are presented in Table 2.

\section{Discussion}

The VITA shade guide analysis presented in Fig. 3 shows that the VITA shade of the control group specimens (C) did not change. The VITA shade of the test1 group specimens (Tx1) did not change, i.e., drilling of microchannels into the tooth did not cause any color change. The VITA shade guide analysis shows that the appearance of the test2 tooth $(\mathrm{Tx} 2)$ is significantly improved after HP-injection during the first $3 \mathrm{~h}$ and does not change for further observations during 24 and $48 \mathrm{~h}$. There is an increase in the VITA shade in the range of 3 to 6 grades depending on the original shade of the tooth.

The CIE $L * a b$ color parameter analysis (see Tables 1 and 2) shows some minor variations for the controls (C) and for the test1 (Tx1) that are, however, within the limits of the

Table $1 \mathrm{CIE} L * a b$ color parameters for the samples from the control $(\mathrm{C})$ and test (Tx1 and Tx2) groups before and 3, 24, and $48 \mathrm{~h}$ after $\mathrm{HP}$-injection through the microchannels drilled by Er:YAG laser radiation.

\begin{tabular}{|c|c|c|c|c|c|c|c|c|c|c|c|c|}
\hline \multirow[b]{2}{*}{ Sample \# } & \multicolumn{3}{|c|}{ Before } & \multicolumn{3}{|c|}{$3 \mathrm{~h}$} & \multicolumn{3}{|c|}{$24 \mathrm{~h}$} & \multicolumn{3}{|c|}{$48 \mathrm{~h}$} \\
\hline & $L$ & $a$ & $b$ & $L$ & $a$ & $b$ & $L$ & $a$ & $b$ & $L$ & $a$ & $b$ \\
\hline $1 \mathrm{C}$ & 61.0 & 5.8 & 17.9 & 61.0 & 5.8 & 17.7 & 60.8 & 5.9 & 17.8 & 61.1 & 5.6 & 17.8 \\
\hline $2 \mathrm{C}$ & 64.5 & 3.9 & 19.1 & 63.9 & 3.9 & 19.5 & 63.9 & 3.7 & 19.6 & 63.2 & 3.8 & 19.7 \\
\hline $3 C$ & 67.8 & 4.0 & 19.2 & 67.5 & 3.9 & 19.9 & 68.1 & 4.0 & 20.1 & 67.7 & 4.2 & 19.6 \\
\hline $4 \mathrm{C}$ & 68.8 & 4.4 & 20.6 & 68.5 & 4.9 & 20.5 & 68.9 & 4.1 & 20.8 & 68.3 & 4.5 & 20.3 \\
\hline $5 C$ & 71.2 & 8.9 & 21.6 & 71.0 & 8.8 & 21.7 & 70.9 & 8.8 & 21.5 & 71.0 & 8.7 & 21.6 \\
\hline $1 \mathrm{Tx} 1$ & 60.0 & 8.3 & 19.0 & 59.2 & 8.1 & 18.8 & 59.6 & 8.2 & 18.9 & 59.6 & 8.2 & 18.7 \\
\hline $2 T x 1$ & 61.1 & 5.7 & 17.8 & 61.0 & 5.7 & 17.6 & 60.5 & 5.5 & 17.8 & 60.9 & 5.6 & 17.7 \\
\hline $3 T \times 1$ & 71.4 & 9.0 & 21.8 & 70.9 & 8.8 & 21.5 & 70.6 & 8.8 & 21.3 & 71.0 & 8.7 & 21.4 \\
\hline $4 \mathrm{~T} \times 1$ & 63.9 & 3.7 & 19.9 & 63.0 & 3.6 & 19.8 & 63.2 & 3.7 & 19.7 & 63.1 & 3.7 & 19.7 \\
\hline $5 \mathrm{~T} \times 1$ & 68.5 & 4.0 & 20.3 & 67.9 & 3.7 & 20.0 & 68.0 & 3.8 & 19.9 & 68.0 & 3.9 & 20.1 \\
\hline $1 T \times 2$ & 60.5 & 8.2 & 18.8 & 68.5 & 5.4 & 13.9 & 72.3 & 4.2 & 12.7 & 72.7 & 4.4 & 12.4 \\
\hline $2 T \times 2$ & 61.2 & 6.3 & 18.9 & 67.5 & 4.4 & 15.9 & 69.8 & 4.0 & 14.7 & 69.8 & 3.9 & 15.4 \\
\hline 3Tx2 & 62.0 & 4.2 & 23.4 & 70.2 & 3.8 & 19.9 & 67.1 & 3.3 & 20.8 & 66.5 & 3.6 & 20.4 \\
\hline $4 \mathrm{~T} \times 2$ & 61.7 & 3.2 & 16.6 & 71.3 & 2.7 & 15.1 & 69.3 & 2.4 & 15.9 & 63.7 & 1.9 & 15.8 \\
\hline $5 T \times 2$ & 68.0 & 3.5 & 19.8 & 70.9 & 3.0 & 15.9 & 76.1 & 3.1 & 15.1 & 79.8 & 2.5 & 15.1 \\
\hline
\end{tabular}


Table 2 Differential color parameters $\Delta(\mathrm{CIE} L * a b)$ for the samples from the control (C) and test (Tx1 and Tx2) groups before and 3,24 , and $48 \mathrm{~h}$ after HP-injection through the microchannels drilled by Er:YAG laser radiation.

\begin{tabular}{|c|c|c|c|c|c|c|c|c|c|c|c|c|}
\hline \multirow[b]{2}{*}{ Sample \# } & \multicolumn{3}{|c|}{ Before } & \multicolumn{3}{|c|}{$3 \mathrm{~h}$} & \multicolumn{3}{|c|}{$24 \mathrm{~h}$} & \multicolumn{3}{|c|}{$48 \mathrm{~h}$} \\
\hline & $\Delta L$ & $\Delta a$ & $\Delta b$ & $\Delta L$ & $\Delta a$ & $\Delta b$ & $\Delta L$ & $\Delta a$ & $\Delta b$ & $\Delta L$ & $\Delta a$ & $\Delta b$ \\
\hline $1 C$ & 0.0 & 0.0 & 0.0 & 0 & 0 & -0.2 & -0.2 & 0.1 & -0.1 & 0.1 & -0.2 & -0.1 \\
\hline $2 C$ & 0.0 & 0.0 & 0.0 & -0.6 & 0 & 0.4 & -0.6 & -0.2 & 0.5 & -1.3 & -0.1 & 0.6 \\
\hline $3 C$ & 0.0 & 0.0 & 0.0 & -0.3 & -0.1 & 0.7 & 0.3 & 0 & 0.9 & -0.1 & 0.2 & 0.4 \\
\hline $4 C$ & 0.0 & 0.0 & 0.0 & -0.3 & 0.5 & -0.1 & 0.1 & -0.3 & 0.2 & -0.5 & 0.1 & -0.3 \\
\hline $5 C$ & 0.0 & 0.0 & 0.0 & -0.2 & -0.1 & 0.1 & -0.3 & -0.1 & -0.1 & -0.2 & -0.2 & 0 \\
\hline Mean (C) & 0.0 & 0.0 & 0.0 & -0.3 & 0.1 & 0.2 & -0.1 & -0.1 & 0.3 & 0.7 & 0.0 & 0.1 \\
\hline$\pm \Delta(\mathrm{C})$ & 0.0 & 0.0 & 0.0 & 0.3 & 0.3 & 0.5 & 0.4 & 0.2 & 0.5 & 0.3 & 0.2 & 0.5 \\
\hline $1 \mathrm{~T} \times 1$ & 0.0 & 0.0 & 0.0 & -0.8 & -0.2 & -0.2 & -0.4 & -0.1 & -0.1 & -0.4 & -0.1 & -0.3 \\
\hline $2 \mathrm{~T} \times 1$ & 0.0 & 0.0 & 0.0 & -0.1 & 0.0 & -0.2 & -0.6 & -0.2 & 0.0 & -0.2 & -0.1 & -0.1 \\
\hline $3 \mathrm{~T} \times 1$ & 0.0 & 0.0 & 0.0 & -0.5 & -0.2 & -0.3 & -0.8 & -0.2 & -0.5 & -0.4 & -0.4 & -0.4 \\
\hline $4 \mathrm{~T} \times 1$ & 0.0 & 0.0 & 0.0 & -0.9 & -0.1 & -0.1 & -0.7 & 0.0 & -0.2 & -0.8 & 0.0 & -0.2 \\
\hline $5 \mathrm{~T} \times 1$ & 0.0 & 0.0 & 0.0 & -0.6 & -0.3 & -0.3 & -0.5 & -0.2 & -0.4 & -0.5 & -0.1 & -0.2 \\
\hline Mean $(\mathrm{T} \times 1)$ & 0.0 & 0.0 & 0.0 & -0.6 & -0.2 & -0.2 & -0.6 & -0.1 & -0.2 & -0.5 & -0.1 & -0.2 \\
\hline$\pm \Delta(\mathrm{T} \times 1)$ & 0.0 & 0.0 & 0.0 & 0.4 & 0.1 & 0.1 & 0.2 & 0.1 & 0.3 & 0.3 & 0.1 & 0.1 \\
\hline $1 \mathrm{~T} \times 2$ & 0.0 & 0.0 & 0.0 & 8.0 & -2.8 & -4.9 & 11.8 & -4.0 & -6.1 & 12.2 & -3.8 & -6.4 \\
\hline $2 \mathrm{~T} \times 2$ & 0.0 & 0.0 & 0.0 & 6.3 & -1.9 & -3.0 & 8.6 & -2.3 & -4.2 & 8.6 & -2.4 & -3.5 \\
\hline $3 T \times 2$ & 0.0 & 0.0 & 0.0 & 8.2 & -0.4 & -3.5 & 5.1 & -0.9 & -2.6 & 4.5 & -0.6 & -3.0 \\
\hline $4 \mathrm{~T} \times 2$ & 0.0 & 0.0 & 0.0 & 9.6 & -0.5 & -1.5 & 7.6 & -0.8 & -0.7 & 2.0 & -1.3 & -0.8 \\
\hline $5 T \times 2$ & 0.0 & 0.0 & 0.0 & 2.9 & -0.5 & -3.9 & 8.1 & -0.4 & -4.7 & 11.8 & -1.0 & -4.7 \\
\hline Mean (Tx2) & 0.0 & 0.0 & 0.0 & 7.0 & -1.2 & -3.4 & 8.2 & -1.7 & -3.7 & 7.8 & -1.8 & -3.7 \\
\hline$\pm \Delta(\mathrm{T} \times 2)$ & 0.0 & 0.0 & 0.0 & 3.2 & 1.3 & 1.6 & 3.0 & 1.8 & 2.6 & 5.6 & 1.6 & 2.6 \\
\hline
\end{tabular}

fluctuations associated with flash power and measuring instrumentation signal instabilities.

A statistical analysis of the differential color parameters $\Delta(\mathrm{CIE} L * a b)$ between the samples from the control (C) group and from the test1 (Tx1) group showed no statistically significant difference between $\Delta(\mathrm{CIE} L * a b)$ determined after 3, 24, and $48 \mathrm{~h}$ ( $p$-value $=0.08$ to $082>0.05)$. Thus, it can be concluded that the drilling of microchannels into the tooth had no statistically significant influence on the CIE $L * a b$ parameters.

A statistical analysis of the differential color parameters $\Delta(\mathrm{CIE} L * a b)$ between the samples from the control (C) group and from the test2 (Tx2) group showed a statistically significant difference between $\Delta(\mathrm{CIE} L * a b)$ determined after 3 , 24 , and $48 \mathrm{~h}(p$-value $=0.01<0.05)$. A statistical analysis of the differential color parameters $\Delta(\operatorname{CIE} L * a b)$ between the samples from test1 (Tx1) group and from the test2 (Tx2) group also showed a statistically significant difference between $\Delta(\mathrm{CIE} L * a b)$ determined after 3,24 , and $48 \mathrm{~h}$ ( $p$-value $=$ $0.01<0.05)$. Thus, it can be concluded that the HP-injection had a statistically significantly influence on the CIE $L * a b$ parameters.

The CIE $L * a b$ color parameter analysis shows that for the test2 samples (Tx2) the $L$ parameter (lightness) is significantly increased due to HP-injection through microchannels. This parameter increases within $3 \mathrm{~h}$ after injection of HP, continues to increase during $24 \mathrm{~h}$, and reaches saturation after $48 \mathrm{~h}$.

It should be noted that the increase in the $L$ parameter (lightness) occurs with a decrease in parameters $a$ (redness) and $b$ (yellowness) as a result of chemical oxidation of pigments which give the red and yellow shades to dentin and tooth. According to Geenwall, ${ }^{30}$ the molecules of these pigments have double bond groups and are organic in origin. ${ }^{31}$ Irreversible discoloration of organic pigments is achieved by reduction of the molecular double bonds. ${ }^{31}$ The main reaction in this case is the impact on the conjugated bonds $(-\mathrm{CH}=\mathrm{CH}-$ ) by $\mathrm{HP}$ or reactive oxygen species with epoque-compound formation. ${ }^{32}$ Under more severe oxidation conditions, the formation of diols or complete $\mathrm{C}-\mathrm{C}$ bond reduction is possible. ${ }^{33}$ 
The bleaching process causes a breakdown of molecular conjugation with the discolored product molecules. A single molecule of HP is consumed for each double bond of organic molecule. The oxidation products do not absorb light in the visible region, therefore, they are colorless. ${ }^{31}$

The above-mentioned alterations in the VITA shade guide and the CIE $L * a b$ color parameters are accompanied by visible variations in the whiteness of the test 2 tooth (Tx2) crown which can be seen in the photographs of the tooth before and after HP-injection (Fig. 2).

The results described above show that a volume of $1.5 \pm 0.1 \mathrm{~mm}^{3}$ of $31 \%$-HP injected into the dentin of a human tooth through microchannels with a diameter of $\sim 200 \mu \mathrm{m}$ and a depth of $\sim 2 \mathrm{~mm}$, which was drilled within the tooth crown using a high-quality beam of Er:YAG laser, is enough for bleaching of the teeth. Other types of lasers, but with a wide-beam diameter at the treatment area of 300 to $1000 \mu \mathrm{m}$, such as Er:YAG, Er:YSGG, or $\mathrm{CO}_{2}$ multimode lasers were used for treatment of dental hard tissue. ${ }^{34-39}$ The cavities created using such lasers with a wide-beam spot are characterized by a low aspect ratio, which is the ratio of cavity depth to cavity diameter, and are intended for traditional fillings. A multimode laser does not allow for formation of laser beams of a sufficiently small diameter for the cutting of the microchannels. The smaller the beam size, the greater the safety level.

Small size beams (microbeams) can form highly accurate cavities whose diameter is significantly smaller than the diameter produced by mechanical instruments (burs). Cavities with a high aspect ratio (microchannels) can also be formed using microbeams.

Another important feature of the laser radiation is the ability to form single spikes (micropulses) with a pulsewidth shorter than the thermal relaxation time of the treated tissue layer. The process of laser hard tooth tissue removal can be significantly improved by controlling the duration of the spikes, their duty cycle, and repetition rate.

Optimization is possible by supplying the laser energy at a particular time corresponding to minimal losses associated with laser beam attenuation by a water irrigation system or tooth debris. The simultaneous use of micropulses and microbeams is known as $\mathrm{M}^{2}$ laser technology. ${ }^{29,40}$ Single-mode $\mathrm{TEM}_{00}$ lasers with a beam propagation ratio close to unity should be used to create these beams. ${ }^{41,42}$ Figure 4 shows a tooth model which was developed to calculate the distribution of HP concentration at the dento-pulpal interface. The model was developed on the basis of the anatomical structure of a tooth as described by Nanci. ${ }^{43}$ The tooth is surrounded by air and its root is composed of dentin and has a cylindrical shape with a diameter of $6.2 \mathrm{~mm}$. The enamel has a thickness of $0.44 \mathrm{~mm}$ and uniformly covers the upper part of the tooth dentin to a height of $10 \mathrm{~mm}$ (similar to a natural tooth). The pulp chamber is cylindrical in shape and has a diameter of $2 \mathrm{~mm}$.

A single channel was drilled at a distance of $5 \mathrm{~mm}$ from the tooth apex, perpendicular to the tooth axis, and its diameter and depth can be varied according to need. The modeling used a depth of $4.4 \mathrm{~mm}$ and a diameter of $0.66 \mathrm{~mm}$, providing a volume of $1.5 \mathrm{~mm}^{3}$ which was equivalent to 5 microchannels, as used in the experimental part of this study. In fact, the surface area of the laser drilled inlet microchannels [see Fig. 1(c)] was larger than that in the model. This difference may have a significant influence on the HP concentration distribution in the vicinity of the channel only within a rather short period of time after injection.

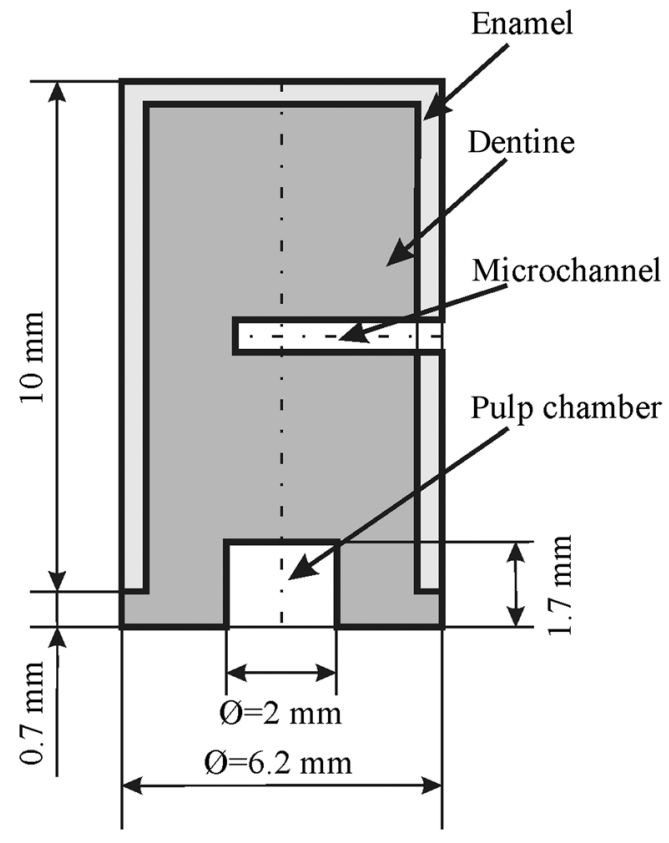

Fig. 4 Tooth model.

As we are really looking for the effect of HP on the entire tooth over a several hours, it can be hypothesized that the abovementioned difference would not alter the results significantly.

The channel could then be filled with an aqueous solution of HP of different concentrations and the injection volume could be varied. The modeling used a concentration of $31 \%-\mathrm{HP}$ and the injected volume was equivalent to the total volume of the channel, i.e., $1.5 \mathrm{~mm}^{3}$.

The concentration of HP was calculated using the following matter diffusion equation: ${ }^{44}$

$\frac{\mathrm{d} C(\vec{r}, t)}{\mathrm{d} t}=D \nabla^{2} C(\vec{r}, t)-\gamma C(\vec{r}, t)$,

where $\vec{r}=(x, y, z)$ is the molecule coordinate, $C$ is the HP concentration, $D$ is the HP diffusion coefficient $\left(\mathrm{m}^{2} / \mathrm{s}\right)$, and $\gamma$ is the absorption factor (due to HP interaction with tissue organic molecules) $\left(\mathrm{s}^{-1}\right)$.

It was assumed that at the beginning of the process, there was no HP in the dentin and enamel, therefore, its concentration in these tissues could be expressed as $C_{e, d}(\vec{r}, t)_{t=0}=0$, and the concentration of HP in the channel as $C_{\mathrm{ch}}(\vec{r}, t)_{t=0}=C_{0}(\vec{r})$, where $C_{0}(\vec{r})$ is the initial concentration of HP in the solution $(31 \%)$. The chemical interaction of the HP with the organic components of the tooth tissues was also not accounted for because of its insignificant effect on HP distribution within tooth tissues, i.e., it was assumed that $\gamma=0$.

The diffusion coefficient of HP in dentin was taken as $D_{d}=$ $3.6 \cdot 10^{-7} \mathrm{~cm}^{2} / \mathrm{s}$ (Ref. 45) and the diffusion coefficient of HP in enamel as $D_{e}=3.6 \cdot 10^{-9} \mathrm{~cm}^{2} / \mathrm{s}^{23}$ The results of numerical modeling of the HP-concentration distribution in a human tooth model are shown in Fig. 5.

Figure 5 shows that the concentration of HP decreases toward the bottom of the channel and does not exceed $2 \%$ at a depth of $2 \mathrm{~mm}$ from the channel inlet. The maximum concentration of HP in the channel is seen immediately after injection [dark red color in Fig. 5(a)]. At $3 \mathrm{~h}$ after injection, the maximum concentration of HP is seen at the entrance to the channel [red 
(a)

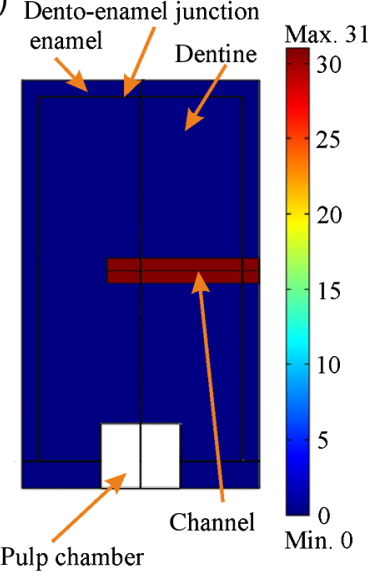

(b)

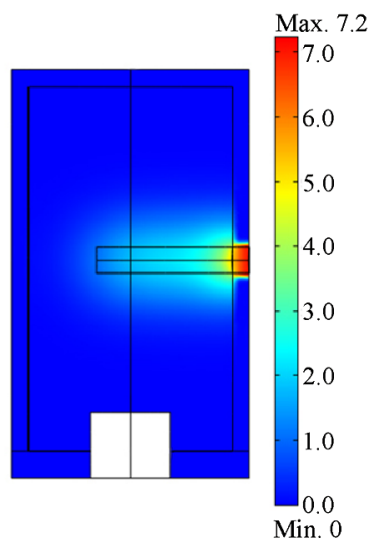

(c)

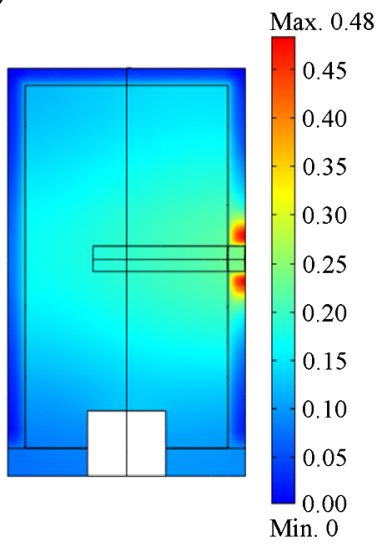

Fig. 5 Numerical modeling of the HP-concentration distribution within tooth tissues calculated using the proposed tooth model: (a) immediately after, (b) $3 \mathrm{~h}$ after, and (c) $48 \mathrm{~h}$ after injection of a $31 \%$-HP solution with a volume of $1.5 \mathrm{~mm}^{3}$ into the channel (volume of the channel is equivalent the volume of 5 microchannels drilled by Er:YAG laser radiation in experiment). The figures on the right of each diagram show the calculated distribution of HP as expressed in a percentage of its concentration. In Fig. 5(a), because the picture shows the concentration of HP at commencement, no difference is seen between the dentin and enamel. However, in the channel cut by laser, it is at its maximum (31\%) and a dark red color.

color in Fig. 5(b)]. At $48 \mathrm{~h}$, the maximum concentration of $\mathrm{HP}$ is seen at the entrance to the channel in the enamel [red color in Fig. 5(c)]. It is distributed more uniformly in dentin and its concentration at the dento-pulpal interface is less than that at the dento-enamel junction.

The calculated concentration of HP in the channel and dento-pulpal interface for varying amounts of HP injected into the channel and absorbed by tooth dentin is shown in Fig. 6. Figure 6(a) shows that HP concentration in the channel decreases over time, but at the dento-pulpal interface it increases, as shown in Fig. 6(b). The results show that an amount of $1.5 \mathrm{~mm}^{3}$ of $31 \%$-HP solution is sufficient to bleach a human incisor in in vitro conditions. At a distance of $2 \mathrm{~mm}$ from the inlet, HP concentration decreases to $10 \%$ in $0.5 \mathrm{~h}$, to $5 \%$ in $1.2 \mathrm{~h}$, and to $1 \%$ in $7 \mathrm{~h}$. It reaches a maximum saturation level at the dento-pulpal interface in $\sim 30 \mathrm{~h}$ and is almost an order of magnitude lower than the safety level as described by Bowles et al. ${ }^{26}$ These results correspond to published in vivo pilot studies. $^{24,40}$

Altshuler et al. ${ }^{40}$ presented results of tooth vitality studies before and after injection of 35\%-HP through microchannels formed by Er; Cr:YSGG laser radiation. The vitality of the dental pulp was assessed using an electric pulp tester. According to Prosvetov et al., ${ }^{46}$ the readings of the pulp tester reach 15 to
$20 \mu \mathrm{A}$ in intact teeth. With inflammation, the readings usually increase to 40 to $80 \mu \mathrm{A}$ depending on the type and intensity of the inflammatory process. In the case of pulpal necrosis, readings are always $>100 \mu \mathrm{A}$. In the study presented by Altshuler et al. ${ }^{40}$ the readings were 0.6 to $6.4 \mu \mathrm{A}$ before laser treatment and immediately after laser treatment there was a slight increase of pulp tester readings but it did not exceed $20 \mu \mathrm{A}$. In a week, the readings returned to the baseline. The H\&E histological analysis ${ }^{40}$ of the intact tooth pulp after internal bleaching using laser microchannels for HP delivery did not show any histological abnormalities in the pulp after bleaching. Tissue necrosis or acute inflammatory infiltration with the accumulation of segmented leukocytes was not found in any of the specimens, ${ }^{40}$ i.e., significant pulpal damage was not observed. From Altshuler et al.'s study, ${ }^{40}$ it can be concluded that the effect on a vital tooth through microchannels formed by Er:YAG laser radiation leads to tooth bleaching which does not produce pulpal damage.

The open microchannels produced by this procedure will need to be restored in an appropriate manner. In principle, it would be possible to use a flowable composite material of which there are many which are currently available on the market. An alternative consideration could be given to a remineralizing technique, but this would be the subject of a further study. (a)

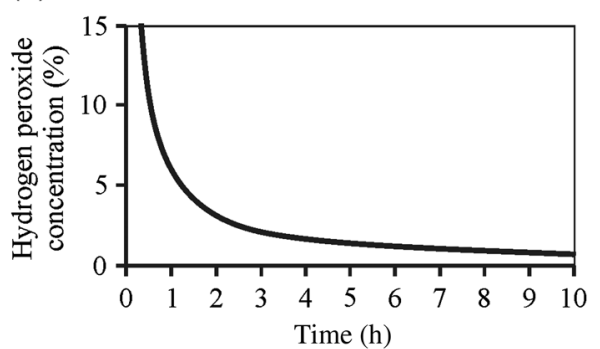

(b)

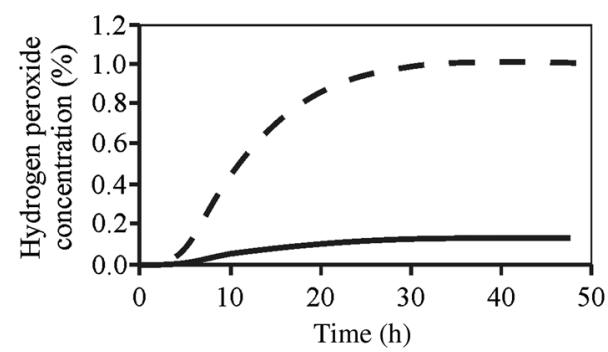

Fig. 6 The calculated concentration of HP on delivery time: (a) in the channel at its central axis, $2 \mathrm{~mm}$ apart from the inlet and (b) in the dentin at the central axis on the dento-pulpal interface. The calculations were made for two different volumes of $31 \%-\mathrm{HP}: 1.5 \mathrm{~mm}^{3}$ (solid line) and $11.5 \mathrm{~mm}^{3}$ (dashed line). 


\section{Conclusions}

A minimally invasive method of controlled intrinsic bleaching of teeth with HP delivered through laser microchannels from the palatal surface of the tooth which can potentially be used for vital tooth bleaching was presented. Laser microchannels were drilled into the tooth crown using multipulse tissue ablation by the radiation of an Er:YAG laser. These microchannels were then used for the delivery of HP directly into the tooth dentin.

The bleaching process is facilitated by the relatively uniform distribution of the bleaching compound into the dentin via free diffusion. Computer simulation showed that the amount of HP necessary for adequate tooth bleaching is well below the safety limit which would cause pulpal damage. The bleaching occurred predominantly during the first $3 \mathrm{~h}$ after HP injection, with a VITA shade increase in the range of 3 to 6 grades depending on the original shade of the tooth.

This result is consistent with the more objective color evaluation using the CIE $L * a b$ color system, where lightness $(L)$ significantly increases after $3 \mathrm{~h}$ of treatment with some saturation at 24 and $48 \mathrm{~h}$, and the two other parameters $a$ (describing behavior in the red-green wavelength range) and $b$ (describing behavior in the yellow-blue wavelength range) decreasing with time.

\section{Disclosures}

No conflicts of interest, financial or otherwise, are declared by the authors.

\section{Acknowledgments}

Valery Tuchin was supported by the Russian Presidential Grant NSh-7898.2016.2 and The National Research Tomsk State University Academic D.I. Mendeleev Fund Program.

\section{References}

1. J. I. Ingle and L. K. Bakland, Eds., Endodontics, 5th ed., BC-Decker, Hamilton, Ontario (2002).

2. S. A. Nathoo, "The chemistry and mechanisms of extrinsic and intrinsic discoloration," J. Am. Dent. Assoc. 128, 6S-10S (1997).

3. A. Watts et al., "Tooth discolouration and staining: a review of the literature," Br. Dent. J. 190(6), 309-316 (2001).

4. D. Dietschi et al., "In vitro colorimetric evaluation of the efficacy of home bleaching and over-the-counter bleaching products," Quintessence Int. 41(6), 505-516 (2010).

5. T. Jiang et al., "Investigation of the effects of $30 \%$ hydrogen peroxide on human tooth enamel by Raman scattering and laser-induced fluorescence," J. Biomed. Opt. 13(1), 014019 (2008).

6. M. Goldberg et al., "Undesirable and adverse effects of tooth-whitening products: a review," Clin. Oral Invest. 14(1), 1-10 (2010).

7. E. M. Bruzell et al., "Side effects of external tooth bleaching: a multicentre practice-based prospective study," Br. Dent. J. 215, E17 (2013).

8. F. F. Demarco et al., "Erosion and abrasion on dental structures undergoing at-home bleaching," Clin. Cosmet. Invest. Dent. 3, 45-52 (2011).

9. M. G. Jorgensen et al., "Incidence of tooth sensitivity after home whitening treatment," J. Am. Dent. Assoc 133(8), 1076-1082 (2002).

10. M. Q. Alqahtani, "Tooth-bleaching procedures and their controversial effects: a literature review," Saudi Dent. J. 26(2), 33-46 (2014).

11. W. Buchalla et al., "External bleaching therapy with activation by heat, light or laser-a systematic review," Dent. Mater. 23(5), 586-596 (2007).

12. C. R. G. Torres et al., "Influence of concentration and activation on hydrogen peroxide diffusion through dental tissues in vitro," Sci. World J. 2013, 193241 (2013).

13. N. U. Wetter et al., "Dental bleaching efficacy with diode laser and LED irradiation: an in vitro study," Laser. Surg. Med. 35(4), 254-258 (2004).
14. L. J. Walsh, "The current status of laser applications in dentistry," Aust. Dent. J. 48(3), 146-155 (2003).

15. H. Jelínková et al., "Laser radiation tooth bleaching," Laser Phys. Lett. 1(22), 617-620 (2004).

16. T. Suemori et al., "A new non-vital tooth bleaching method using titanium dioxide and $3.5 \%$ hydrogen peroxide with a $405-\mathrm{nm}$ diode laser or a halogen lamp," Laser Phys. Lett. 5(6), 454-459 (2008).

17. T. Suemori et al., "Effects of light irradiation on bleaching by a $3.5 \%$ hydrogen peroxide solution containing titanium dioxide," Laser Phys. Lett. 5(5), 379-383 (2008).

18. J. O. Calatayud et al., "Clinical efficacy of a bleaching system based on hydrogen peroxide with or without light activation," Eur. J. Esthetic Dent. 5(2), 216-224 (2010).

19. B. C. Borges et al., "Preliminary clinical reports of a novel night-guard tooth bleaching technique modified by casein phosphopeptideamorphous calcium phosphate (CPP-ACP)," Eur. J. Esthetic Dent. 6(4), 446-453 (2011)

20. R. Ganesh et al., "Comparison of the bleaching efficacy of three different agents used for intracoronal bleaching of discolored primary teeth: An in vitro study," J. Indian Soc. Pedod. Prev. Dent. 31(1), 17-21 (2013).

21. K. M. Ghalili et al., "Clinical study of the safety and effectiveness of a novel over-the-counter bleaching tray system," J. Clin. Cosmet. Invest. Dent. 6, 15-19 (2014).

22. M. A. M. Sulieman, "An overview of tooth-bleaching techniques: chemistry, safety and efficacy," Periodontology 48(1), 148-169 (2008).

23. A. L. Ubaldini et al., "Hydrogen peroxide diffusion dynamics in dental tissues," J. Dent. Res. 92(7), 661-665 (2013).

24. G. B. Altshuler et al., "New method for intrinsic tooth whitening using laser microperforation," in Int. Association for Dental Research Annual Conf., Miami, Florida (2009).

25. A. V. Belikov et al., "New method for intrinsic whitening of vital teeth using Er, Cr:YSGG laser microperforation: first in vivo cases," in Academy of Laser Dentistry 16th Annual Conf., Las Vegas, Nevada (2009).

26. W. H. Bowles et al., "Vital bleaching: the effects of heat and hydrogen peroxide on pulpal enzymes," J. Endodont. 12(3), 108-112 (1986).

27. W. M. Bengel, "Digital photography and the assessment of therapeutic results after bleaching procedures," J. Esthet. Restor. Dent. 15(s1), S21-S32 (2003)

28. ISO/TC, International Standard ISO 11146-1:2005(E), Lasers and Laser-Related Equipment-Test Methods for Laser Beam Widths, Divergence Angles and Beam Propagation Ratios-Part 1: Stigmatic and Simple Astigmatic Beams, ISO, Santa Clara (2005).

29. G. B. Altshuler et al., "Hard tissue ablation," in Handbook of Biophotonics, Vol. 2, Photonics for Health Care, J. Popp et al., Eds., Wiley-VCH Verlag GmbH \& Co. KgaA, Weinheim (2011).

30. L. Greenwall, Ed., Bleaching Techniques in Restorative Dentistry: an Illustrated Guide, Martin Dunitz Ltd., New York (2001).

31. H. Zollinger, Color Chemistry: Syntheses, Properties, and Applications of Organic Dyes and Pigments, Wiley, Weinheim (2003).

32. R. C. Neuman, Jr., "Organic chemistry," University of California (2009), http://web.chem.ucsb.edu/ neuman/orgchembyneuman/BookContents .html (July, 2016).

33. V. K. Ahluwalia and R. K. Parashar, Organic Reaction Mechanisms, Alpha Science International, Oxford (2007).

34. D. J. Coluzzi and R. A. Convissar, Atlas of Laser Applications in Dentistry, Quintessence Publishing Co., London (2007).

35. T. Dostalova et al., "Evaluation of depth and profile cavity after laser ablation with different energy of Er:YAG laser radiation," Proc. SPIE 2623, 88-93 (1996).

36. Y. Nishimoto et al., "Effect of pulse duration of Er:YAG laser on dentin ablation," Dent. Mater. J. 27(3), 433-439 (2008).

37. R. Hibst et al., "Experimental studies of the application of the Er:YAG laser on dental hard substances: I. Measurement of the ablation rate," Laser. Surg. Med. 9(4), 338-344 (1989).

38. K. Stock et al., "Comparison of Er:YAG and Er:YSGG laser ablation of dental hard tissues," Proc. SPIE. 3192, 88-95 (1997).

39. R. de F. Z. Lizarelli et al., "Comparative ablation rate from a Er: YAG laser on enamel and dentin of primary and permanent teeth," Laser Phys. 16(5), 849-858 (2006).

40. G. B. Altshuler et al., "'M2 laser technology” and its application for dental bleaching," Innov. Dent. 1, 44-54 (2010). 
41. J. Meister et al., "Influence of the spatial beam profile on hard tissue ablation Part I: multimode emitting Er:YAG lasers," Laser. Med. Sci. 18(2), 112-118 (2003).

42. J. Meister et al., "Influence of the spatial beam profile on hard tissue ablation, Part II: pulse energy and energy density distribution in simple beams," Laser. Med. Sci. 19(2), 112-118 (2004).

43. A. Nanci, Ten Cate's Oral Histology: Development, Structure, and Function, 6th ed., Mosby, London (2003).

44. E. L. Cussler, Diffusion: Mass Transfer in Fluid Systems, Cambridge University Press, Cambridge (2009).

45. N. Trunina et al., "Dentinal permeation modeling," Proc. SPIE 6791, 67910T (2008).

46. R. S. Prosvetov et al., "Features of electroexcitability of a pulp of teeth at students from different regions of the world," Stomatologiia 4, 26-27 (2013).

Gregory Altshuler is a director of medical products of IPG Photonics Corporation, Oxford Massachusetts, USA, a leading researcher, and manufacturer of fiber and diode lasers. He has published more than 200 scientific papers, chapters of 6 books, and holds 141 patents. His research interests include research and development new technologies and laser platforms for laser medicine, novel technologies based on new generation diode lasers, fiber lasers, and diode pumped solid state lasers.

Andrey Belikov is a professor in the Laser Technologies and Systems Department of Saint-Petersburg National Research University of Information Technologies, Mechanics and Optics (ITMO University, Russia). He participated in more than 20 international congresses, 5 invited lectures, and he has published more than 150 scientific papers. His research interests include lasers, biomedical optics, laser-tissue interaction, and laser optics.

Alexei Skrypnik is an associate professor in the Laser Technologies and Systems Department of Saint-Petersburg National Research University of Information Technologies, Mechanics and Optics (ITMO University, Russia). He participated in more than 18 international congresses and has published more than 100 scientific papers. His research interests include biomedical optics, laser-tissue interaction, and nanotechnologies.
Felix Feldchtein received his $\mathrm{PhD}$ in quantum electronics from the Institute of Applied Physics of the Russian Academy of Sciences. $\mathrm{He}$ is a medical device consultant. He has more than 20 years of academic experience involving a variety of disciplines, including lasers, nonlinear optics, laser-tissue interaction, optical spectroscopy, and imaging. For the last 20 years, the main focus is in the development and commercialization of medical devices in the area of laser medicine.

Alexandra Pushkareva is an associate professor in the Laser Technologies and Systems Department of Saint-Petersburg National Research University of Information Technologies, Mechanics and Optics (ITMO University, Russia). She participated in more than 8 international congresses. Her research interests are biomedical optics, physics of interaction of light with materials, and medical laser applications. She has published more than 25 scientific papers.

Ksenia Shatilova is an associate professor in the Laser Technologies and Systems Department of Saint-Petersburg National Research University of Information Technologies, Mechanics and Optics (ITMO University, Russia). She participated in 2 international projects (optics for medicine), more than 10 international congresses, and has published more than 40 scientific papers. Her research interests include biomedical optics, physics of interaction of light with materials, and medical laser applications.

Igor Cernavin is a prosthodontist and an honorary senior fellow at the University of Melbourne, School of Dentistry, Faculty of Medicine, Dentistry and Health Sciences, Melbourne, Australia, and the Australian representative of World Federation of Laser Dentistry (WFLD).

Valery Tuchin is a professor and chairman of optics and biophotonics at Saratov State University. He is also the head of laboratory at the Institute of Precision Mechanics and Control, Russian Academy of Science. His research interests include biophotonics, tissue optics, laser medicine, tissue optical clearing, and nanobiophotonics. He is a member of SPIE, OSA, and IEEE. He is a fellow of SPIE and has been awarded Honored Science Worker of Russia, SPIE Educator Award, and FiDiPro (Finland). 